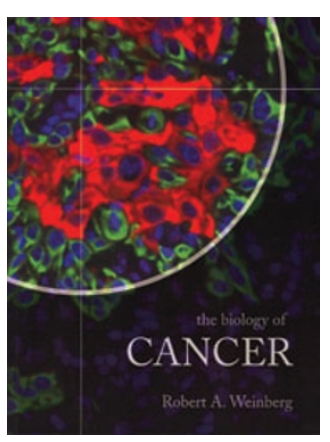

\section{Secrets of a killer}

\section{The Biology of Cancer}

By Robert Weinberg

Garland Science, $2006 \bullet \$ 89.99 / £ 44.00$

Reviewed by Alice T. Hawley and

Pier Paolo Pandolfi

The development of modern molecular biology in the twentieth century yielded outstanding advances in our understanding and treatment of cancer. Cracking the DNA code, and the subsequent advent of molecular cloning, allowed biologists to start unravelling human disease on a molecular level. Robert Weinberg, a prominent cancer biologist who has made key contributions to this effort, has recently written a textbook, The Biology of Cancer, in which he elegantly guides students through the history of this revolution, the scientific thinking that enabled it and the biological principles that were discovered. The text is the product of both Weinberg's intellect and that of the numerous leading biological researchers he consulted to ensure the accuracy of the content. The breadth of the topics covered, the clear writing style, the logical organization and the beautiful figures make The Biology of Cancer a classic text for any biologist interested in cancer.

The structure and style of the book succeed in creating an accessible introduction to cancer biology. Weinberg's conversational writing style is sure to be understandable to students with little or no biology background (although the text does assume some understanding of molecular biology, which is not discussed in detail). It is clear that Weinberg is aware of his audience at all times; for example, he includes the phonetic pronunciations for gene names such as $\operatorname{Src}$ or $\mathrm{Shc}$ - an attention to detail that is likely to spare new students from embarrassment in classes or presentations.

The first four chapters of the book mainly take a historical perspective and enumerate the key discoveries on which modern cancer biology is built. This discussion begins with Mendel and classical genetics, and proceeds to the beginning of modern cancer biology when tumour-inducing viruses were discovered to harbour oncogenes that can also exist naturally in the human genome. Drawing the reader in, Weinberg humanizes this historical account with quotes and brief descriptions of the scientists and circumstances surrounding pivotal discoveries. Importantly, he spells out the thinking and the experimental assays that led to

Alice Hawley and Pier Paolo Pandolfi are at the Beth Israel Deaconess Medical Center and Harvard Medical School, 77 Avenue Louis Pasteur, Boston, MA 02115, USA.

email:ahawley@bidmc.harvard.edu;ppandolf@bidmc.harvard.edu these discoveries, a necessity for a textbook that seeks to train new scientists. Throughout the text, Weinberg highlights the fact that cancer biology requires the convergence of molecular biology, cell biology, biochemistry and genetics. The contributions from these disciplines are illustrated with beautiful colour micrographs, diagrams, schematics of protein structures, and experimental data.

The next two chapters discuss the growth factor receptors and signalling pathways that are frequently altered in human cancer. This section is an especially valuable resource for new biology students, who may be overwhelmed by the complicated signalling diagrams that they encounter in their studies. Weinberg dissects many critical pathways (for example, Ras-Raf-Mek-Erk) step by step, allowing the reader to appreciate both the molecular mechanisms underlying these molecular switches and the cell biological changes that these pathways bring about. Understandably, this discussion is by no means comprehensive, because it focuses on a relatively small number of well-studied pathways. This allows Weinberg to illustrate the principles of signal transduction in a manner comprehensible to students with no background in this field and should allow students to understand the logic presented in complex signalling diagrams (such as those in the included poster), transforming them from anxiety-inducers to useful learning and memory tools. This section, however, could have benefited from a description of the experimental techniques that are used to dissect the linear relationships shown in these pathways and may have been omitted for simplicity.

In chapters 7-9 Weinberg spells out the principles of tumour suppression, including descriptions of the cell cycle and apoptosis machinery; these are presented from the point of view of a cancer biologist, for example by emphasizing the role of extracellular signals in regulating entry to the cell cycle. Having laid the foundation for a more sophisticated discussion of cancer progression, Weinberg then articulates our current understanding of tumour progression, with a focus on the role of clonal evolution in response to cell-autonomous and non-cell-autonomous selective pressures in chapters 10 and 11 . After describing the role of DNA repair genes as master guardians of the genome, Weinberg lays out our current knowledge of the role of the stroma in tumour progression, invasion and metastasis. The book concludes by discussing modern therapeutic strategies for cancer, including both immunology-based tumour vaccines and traditional small-molecule therapies.

An additional strength of the book is the multimedia CD that contains JPEGs and PowerPoint files of the colour figures from the book; this feature is not only a great classroom tool but also a valuable resource for any researcher who constructs professional presentations. The CD also includes six movies illustrating key concepts, such as the structural basis for Ras activation. However, all the movies were originally published as supplementary material for Molecular Biology of the Cell. Finally, the disc also includes six audio lectures by Dr. Weinberg himself that can be downloaded to your mp3 player or played on the computer. Thus, The Biology of Cancer is not only a great read but also an outstanding learning tool. 